\title{
Von Lefort zu Mouffe. Populismus als Moment und Grenze radikaler Demokratie
}

\section{Seongcheol Kim}

Angenommen: 3. Mai 2021 / Online publiziert: 10. Juni 2021

(C) Der/die Autor(en) 2021

Zusammenfassung In diesem Beitrag werden Chantal Mouffes jüngste Interventionen für den Linkspopulismus zum Anlass für eine theoretische Reflexion über das Spannungsverhältnis zwischen Populismus und radikaler Demokratie genommen. Im Lichte der radikalen Demokratietheorien Claude Leforts, Jacques Rancières, Ernesto Laclaus und Chantal Mouffes, der Populismustheorie Laclaus sowie der einschlägigen Rezeption wird argumentiert, dass der Populismus sowohl konstitutives Moment als auch Grenze eines radikalen Demokratieverständnisses bildet, was sich insbesondere in der Akzentverschiebung von Autonomie zu Repräsentation bei Laclau verdichtet und als Organisationsfrage bei Mouffe hervortritt. Damit öffnet sich nicht zuletzt eine empirische Forschungsperspektive zur Untersuchung des Verhältnisses von Populismus und radikaler Demokratie, die zum Schluss exemplarisch aufgegriffen und anhand einer Unterscheidung zwischen Bewegungsparteien und Volksparteien neuen Typs veranschaulicht wird.

Schlüsselwörter Demokratietheorie $\cdot$ Parteien $\cdot$ Populismus $\cdot$ Radikale Demokratie $\cdot$ Soziale Bewegungen 


\section{From Lefort to Mouffe: Populism as moment and limit of radical democracy}

Abstract This article takes up Chantal Mouffe's recent interventions in support of left populism as a starting point for a theoretical reflection on the tension between populism and radical democracy. In light of the radical democratic theories of Claude Lefort, Jacques Rancière, Ernesto Laclau, and Chantal Mouffe, Laclau's theory of populism as well as the relevant reception, it is argued that populism emerges as both a constitutive moment and a limit of a radical understanding of democracy, which crystallizes in the shift in accent from autonomy to representation within Laclau's work in particular and comes to the fore as a question of organization for Mouffe. The article thus opens up an empirical research perspective for examining the relationship between populism and radical democracy, taking up a series of examples at the end and proposing a distinction between movement parties and people's parties of a new type.

Schlüsselwörter Democratic theory $\cdot$ Political parties $\cdot$ Populism $\cdot$ Radical democracy $\cdot$ Social movements

\section{Einleitung}

Zehn Jahre nach dem globalen Aufkommen der sog. Platzbewegungen - vom Arabischen Frühling bis hin zu Indignados und Occupy - sind zwei Politikformen zunehmend in den Mittelpunkt politikwissenschaftlicher und -theoretischer Debatten gerückt: Populismus und radikale Demokratie. In ihrem neuesten Buch plädiert Chantal Mouffe für einen „linken Populismus“ mit dem Ziel einer „Radikalisierung der Demokratie“ und folgt damit einer bestimmten gegenwartsdiagnostischen Tendenz innerhalb der radikalen Demokratietheorie und -forschung, vor dem Hintergrund der Protestmobilisierungen des vergangenen Jahrzehnts die möglichen Affinitäten - bzw. „Synergien“, wie Mouffe es nennt - zwischen radikaldemokratischer und (links-)populistischer Politik hervorzuheben (vgl. Errejón und Mouffe 2015; Katsambekis 2016; Kioupkiolis 2016; Gerbaudo 2017a, b). Auffällig unterbelichtet bleiben dabei die Spannungen zwischen Populismus und radikaler Demokratie, was umso bemerkenswerter erscheint, da Mouffe und Ernesto Laclau in ihrem gemeinsamen Frühwerk eine ausgefeilte Theorie der radikalen Demokratie entwickelt hatten. Grundsätzlich lässt sich innerhalb der zeitgenössischen radikalen Demokratietheorie eine Spaltung zwischen „ontologies of abundance“ und „ontologies of lack“ identifizieren (Tønder und Thomassen 2005): Während Bewegungen wie Occupy und 15-M für die einen auf eine radikal nicht-hierarchische, gar post-repräsentative Form von Politik verwiesen (vgl. Graeber 2012; Hardt und Negri 2012; Lorey 2012; Sitrin und Azzellini 2014; Tsomou 2017), stellten sie für die anderen eine Art Populismus von unten dar, der die unerfüllten Versprechen des demokratischen Systems im Namen eines Volkssubjekts reklamierte und damit auch den gegenhegemonialen Anspruch erhob, die Demokratie anders zu organisieren und zu praktizieren (vgl. Gerbaudo 2017a, b), ohne sich dabei auf das institutionalisierte Spiel von Wahlen 
und Parlamenten einzulassen (vgl. Errejón und Mouffe 2015). Vor diesem Hintergrund plädiert Mouffe (2018, S. 30) nämlich für einen Linkspopulismus, der den Schritt in die Institutionen wagt, lässt aber die Frage unberücksichtigt, wie das radikaldemokratische Moment dieser Bewegungen dabei erhalten bleiben kann - nicht zuletzt im Sinne der „Autonomie“ demokratischer Kämpfe, die in der radikalen Demokratietheorie Laclaus und Mouffes eine zentrale Rolle gespielt hatte. Insofern bildet Mouffes Plädoyer die Spitze eines Eisbergs, indem eine bestimmte theoretische Haltung zu Populismus und radikaler Demokratie zwar zugespitzt vorkommt, aber die tiefere Problemlage weitgehend verborgen bleibt.

Im vorliegenden Beitrag werden Mouffes jüngste Interventionen zum Anlass für eine tiefergehende Reflexion über das Verhältnis von radikaler Demokratie und Populismus genommen, und zwar sowohl im Hinblick auf den ,Kanon“ der radikalen Demokratietheorie als auch an empirischen Fallbeispielen von Organisationspraktiken an der Schnittstelle zwischen Bewegung und Partei, die den Bogen zurück zu Mouffes Interesse an linkspopulistischer Strategie spannen. Fasst man Populismus als „Diskursstrategie“ zur „Mobilisierung der ,Benachteiligten“ gegen ,die an der Macht“" (Mouffe 2018, S. 20), stellt sich zunächst die Frage, wie sich Populismus im Spiegel unterschiedlicher radikaler Demokratietheorien einordnen lässt. Kennzeichnend für die radikaldemokratische Denktradition des ,lack“, die im Mittelpunkt dieser Abhandlung steht, ist die Idee, dass jeder Versuch, Gesellschaft zu gründen, konstitutiv mangelhaft ist und dadurch ein unabschließbares politisches Ringen um konkurrierende Gründungsversuche hervorbringt; hierbei kann der Populismus durch die Berufung auf demokratische Gründungsversprechen wie die Volkssouveränität einen geradezu unverzichtbaren Impuls zur Radikalisierung der Demokratie geben, gleichzeitig aber auch die Gefahr bergen, die Kontingenz und Unvollkommenheit des Sozialen zu verdecken. Die verschiedenen Spielarten radikaler Demokratietheorie legen somit eine unauflösbare, aber auch produktive Spannung zwischen Populismus und radikaler Demokratie nahe, wie die folgenden Skizzen unter Rückgriff auf die einschlägige Rezeption bekräftigen: Populismus bildet ebenso ein konstitutives Moment wie eine Grenze radikaler Demokratie und verspricht sowohl die Aktivierung des „symbolischen Dispositivs“ der Demokratie als auch die Möglichkeit von dessen totalitärer Aufhebung (mit Lefort), sowohl Politik als auch Polizei (mit Rancière), sowohl die horizontale Ausweitung „demokratischer Kämpfe“ als auch vertikale hegemoniale Führung (mit Laclau/Mouffe). In einigen von Mouffes Interventionen wird dieses Spannungsverhältnis wiederum auf die organisationspolitische Ebene verlagert und lokalisiert: Im Linkspopulismus komme es darauf an, so Mouffe, eine ,neue politische Organisationsform“ zu erfinden, die die unterschiedlichen demokratischen Forderungen sowohl in „horizontalistische Ausdrucksformen“ einbinden als auch vertikal um den Namen eines Volkssubjekts zusammenführen müsse (Errejón und Mouffe 2015, S. 113f.). Damit stellt sich bei der empirischen Erforschung des Verhältnisses von radikaler Demokratie und Populismus zunehmend die Frage nach den Organisationsformen und -praktiken von Parteiformationen, die sowohl an autonome Kämpfe anknüpfen als auch auf die populistische Anrufung eines Volkssubjekts setzen, was sich wiederum mit der diskurs- und hegemonietheoretischen Begrifflichkeit Laclaus und Mouffes analytisch nachzeichnen lässt. Im Anschluss an die theoretischen Überlegungen wird anhand einiger empirischer Fall- 
beispiele ein erster Versuch der konzeptuellen Einordnung unternommen und eine Unterscheidung zwischen Bewegungsparteien und Volksparteien neuen Typs vorgeschlagen, die auf zwei unterschiedliche Arten der organisatorischen Aushandlung von Autonomie und Repräsentation verweist. Somit hofft der vorliegende Aufsatz, einen Beitrag zu einem theoretischen und empirischen Zusammendenken dessen, was Mouffe einen ,populistischen Moment“ im Nachgang der Platzbewegungen nennt, leisten zu können.

\section{Populismus im Spiegel der radikalen Demokratietheorie Leforts: Zwischen „Ideologie der Demokratie“" und Totalitarismus}

Die Demokratietheorie Claude Leforts rückt die radikale Kontingenz und Unvollständigkeit der Demokratie in den Vordergrund und ist für weitere Spielarten radikaler Demokratietheorie in dieser Hinsicht richtungsweisend. Marchart (2007, S. 86) betont in seiner postfundamentalistischen Lesart den Status Leforts als ,contingency theorist“, der die Demokratie als die erste Gesellschaftsform auffasst, ,die die Unbestimmtheit in ihre Form aufnimmt und erhält" (Lefort 1990, S. 291). In radikalem Gegensatz zur absoluten Monarchie wird in der Demokratie demnach der Ort der Macht von einer transzendentalen Grundlage (wie z. B. dem Gottesgnadentum) entbunden und zur „Leerstelle“ gemacht, die einem „Konflikt der kollektiven Willensäußerungen unterworfen“" und damit grundsätzlich zu dessen kontingenten und temporären Ergebnis reduziert wird (Lefort 1990, S. 293 f.). Damit wird der doppelte Körper des Königs - der sterbliche Körper der königlichen Person einerseits und deren vermeintlich unsterbliche Körper andererseits, der als mystisch-symbolischer Letztgarant für die Einheit der Domäne fungiert - aufgebrochen und die konstitutive Unvollständigkeit bzw. Körperlosigkeit von Gesellschaft zur Schau gestellt, die sich nicht mehr auf eine den „Konflikt der kollektiven Willensäußerungen“ transzendierende Einheit berufen und sich stattdessen lediglich auf die Möglichkeit von Konflikt als „,negative[n] Grund von Gesellschaft“ (Marchart 2010, S. 137) stützen kann. Hierin besteht auch die Besonderheit der Demokratie als der ,geschichtliche[n] Gesellschaft schlechthin“ (Lefort 1990, S. 293), die ihre eigene politische Verfasstheit und damit letztliche Grundlosigkeit sichtbar macht: Einerseits gilt für jede Gesellschaft, dass sie als solche erst durch einen politischen Akt der Formund Sinngebung instituiert wird - und dass die Tatsache, ob „die Politik“ als Teilsystem einer sich immer weiter ausdifferenzierenden Gesellschaftsformation oder als exklusive Herrschaftsdomäne eines Fürsten betrachtet wird, wiederum auf den Gründungsakt des Politischen zurückgeht; andererseits räumt die Demokratie als einzige Gesellschaftsform dem Politischen explizit die Funktion einer Gründungsinstanz ein und erkennt damit ihre letztliche Grundlosigkeit an, indem sie durch den institutionalisierten Konflikt von Willensäußerungen ihre eigenen Konturen immer wieder neu bestimmen lässt.

Dabei implizieren Leforts Überlegungen nicht, dass die Demokratie jegliche Vorstellung einer Einheit der Gesellschaft verwirft; vielmehr wird diese zum Gegenstand einer konflikthaften Aushandlung, anstatt sich auf eine vorbestimmte und für allemal gültige Substanz berufen zu können. Indem die Macht in der Demokratie zum 
Objekt permanenter Auseinandersetzung wird, wird sie eben nicht abgeschafft, sondern nimmt die umso konstitutivere Funktion ,,jene[r] Instanz“ ein, „kraft derer die Gesellschaft sich in ihrer Einheit erfaßt und sich in Zeit und Raum auf sich selbst bezieht" (Lefort 1990, S. 293). Den Ort der Macht als Leerstelle zu beachten, heißt aber, dass jeder Macht- und Einheitsanspruch nur partiell und eingegrenzt sein kann, sich also dem „Konflikt der kollektiven Willensäußerungen“ stellen muss und diese wiederum nicht (etwa im Namen eines Ewigkeitsanspruchs) außer Kraft setzen darf. Somit wird die Macht grundsätzlich ,circumscribed and localized in society at the same time as being an instituting moment“ (Lefort 1986, S. 305). Kennzeichnend für den „Totalitarismus“ hingegen ist ,die Vorstellung einer homogenen und für sich selbst durchsichtigen Gesellschaft, des einen Volkes“ (Lefort 1990, S. 287), das angeblich über dem Konflikt unterschiedlicher Willensäußerungen steht und - wie im absolutistischen Imaginären, allerdings bei gleichzeitiger Berufung auf das demokratische Gründungsprinzip der Volkssouveränität - in der Person eines Führers restlos verkörpert wird. Totalitarismus zeichnet sich also durch die Verneinung von Kontingenz und sozialer Teilung aus: Durch den exklusiven Anspruch auf ,das Volk“ - das vermeintlich nur in einer möglichen Form repräsentiert werden kann - wird der Streit um dessen Konstituierung kurzgeschlossen und damit auch das ,symbolische Dispositiv“ der Demokratie (Lefort 1992) ausgehebelt.

In der liberalen politischen Theorie gibt es mitunter die fragwürdige Tendenz, Populismus in der Nähe des Totalitarismus im Lefort'schen Sinne zu verorten (Abts und Rummens 2007; Müller 2014, 2016). In der Theorie Jan-Werner Müllers avanciert der Alleinvertretungsanspruch gegenüber einem einzig wahren, Volk‘ zum Definitionskern des Populismus, womit Letzterer in die Nähe eines bloßen Synonyms dessen rückt, was Lefort Totalitarismus nennt. Müller (2014, S. 488) selbst spricht von einer „Affinität“ seiner Populismusdefinition zum Lefort'schen Totalitarismusbegriff im Sinne von ,the image of a pre-procedural people, as represented by a party or a single leader [...] seek[ing] to occupy democracy's empty space of power." Allerdings geht aus Leforts Theorie nicht hervor, dass die Berufung auf ,das Volk“ als solche exklusiv-totalisierende Implikationen hat; vielmehr besteht gerade der demokratische Spagat darin, die Einheit der Gesellschaft und die Konstituierung des ,Volkes‘ zum Gegenstand einer konflikthaften Aushandlung zu machen, ohne dass der Fortbestand dieses institutionell geregelten Prozesses dadurch subvertiert wird. Fasst man Populismus nicht von vornherein als exklusiv-totalisierend, sondern im breiteren Sinne als die Berufung auf ein ,Volk' gegenüber einer instituierten Macht, liegt es sogar nahe, dass dieser aus dem demokratischen Kampf von Willensäußerungen nicht wegzudenken ist, da gerade die Demokratie eine unüberbrückbare Lücke voraussetzt zwischen dem ,Volk', das im symbolischen Dispositiv der Demokratie als machtgebende Instanz anerkannt wird, und der Macht, die niemals identisch mit dem, Volk' sein darf, auf das sie sich berufen muss. So gesehen wäre jeder Populismus zunächst ein Ausdruck von Demokratie, der die konstitutive Spaltung zwischen Volk und Macht politisch artikuliert und damit auch die Teilung des Sozialen sichtbar macht; ob dabei auch noch das totalisierende Bild eines einzig wahren und exklusiv repräsentierbaren Volkes als „substantielle Realität[]“ (Lefort 1990, S. 295) vertreten und damit ein Alleinvertretungsanspruch erhoben wird, wäre 
die Frage, die darüber entscheidet, ob das symbolische Dispositiv der Demokratie in derselben Bewegung untergraben wird, wie es aktiviert wird.

Nach Margaret Canovans Lefort-Lektüre nimmt der Populismus in dieser Hinsicht eine doppelte Stellung als konstitutiver, aber auch ambivalenter Bestandteil der Demokratie ein: Einerseits bildet er die ,ideology of democracy“ schlechthin (Canovan 2002), die ,democracy's promise of power to the people“ einlösen will (Canovan 1999, S. 2); andererseits verweist er auf die Möglichkeit einer totalitären Außerkraftsetzung des demokratischen Dispositivs im Lefort'schen Sinne, denn ,,its craving for transparency in the exercise of popular sovereignty must harbour totalitarian possibilities“ (Canovan 2002, S. 41). Insofern bildet Populismus sowohl eine wiederkehrende Möglichkeit (,,a perennial possibility“) innerhalb der Demokratie, die in deren symbolischen Dispositiv, im Gründungsversprechen bereits angelegt ist, als auch eine mögliche Grenze, die aus dem demokratischen Spiel der Kontingenz heraus in den kontingenzleugnenden Pol des Totalitarismus umschlagen kann. Fasst man das Problem des Totalitarismus nicht zuletzt als Verneinung der Kontingenz, so lässt sich weiterführen, dass die totalitäre bzw. autoritäre Gefahr insbesondere aus jenen Spielarten des Populismus hervorgeht, die die populistische Frontstellung Volk/ Macht mit einer reduktionistischen Logik der Unterstellung ,ein[es] präexistente[n] und homogene[n] Volk[es]“ (Möller 2017, S. 247) verknüpfen und damit zur Verneinung der Möglichkeit abweichender Volkskonstruktionen tendieren. Gerade dieses Zusammenspiel von Populismus und Reduktionismus - das für rechtspopulistische Diskurse kennzeichnend ist (vgl. Kim 2017) - legt eine erhöhte Gefahr des Totalitarismus nahe, indem ,das Volk‘ als uneingelöstes Subjekt der Demokratie nicht nur einem Machtblock gegenübergestellt, sondern auch apriorisch an einem ,transcendental signified“ festgemacht wird (Stavrakakis et al. 2017) - sei dies etwa ethnischer, kultureller und/oder religiöser Natur -, um das Bild eines einzig möglichen Volkes zu erzeugen. Dass dies aber nur auf bestimmte Spielarten des Populismus zutrifft, verweist wiederum auf die grundlegende Ambivalenz zwischen Populismus und (radikaler) Demokratie; fasst man Populismus von vornherein als ausschließlich autoritär, geht die Ambivalenz, wohl aber auch der besondere Wert des Begriffs verloren (vgl. Stavrakakis und Jäger 2018).

\section{Populismus im Spiegel der radikalen Demokratietheorie Rancières: Zwischen Politik der Anteillosen und Polizei}

In der Demokratietheorie Jacques Rancières lässt sich ein besonders zugespitzter Kontingenz- und Unvollständigkeitsgedanke auffinden, der allerdings die konstitutive Leerstelle der Demokratie beim Volk verortet. „Es ist zuerst das Volk, und nicht der König, das einen doppelten Körper hat", antwortet Rancière (2008, S. 25) auf Lefort - und das nicht erst seit der demokratischen Revolution, sondern als ,,konstitutive Gegebenheit der Politik“. Denn die Logik jeder Herrschaftsgewalt beruht demnach auf der Zählung und Erfassung dessen, was in der Ordnung der Dinge als sichtbar, hörbar und vernehmbar gelten darf - eine Logik, die Rancière (2002, S. 39) „Polizei“ nennt, die „,nach ihren ,Eigentümlichkeiten“ die Körper auf ihren Platz und an ihre Funktionen verweist“ -, während die „Politik“ hingegen das Moment der 
Unterbrechung polizeilicher arche bildet, indem die Anteillosen bzw. Ungezählten der Zählung ihren Anteil reklamieren und damit die gesamte Logik der Zählung radikal infrage stellen. Die Politik tritt also als Grenze der Objektivität und damit auch als Moment radikaler Kontingenz in Erscheinung, wo der polizeiliche Versuch der Sinnfixierung von einem Realen im Lacanschen Sinne überströmt wird nämlich vom „Volk“ als „leere[m], supplementäre[m] Teil“, dessen Abstand zum verkörperten Sinnlichen sichtbar geworden ist (Rancière 2008, S. 24). „Das Volk“ als „das Subjekt der Demokratie“ ist für Rancière (2008, S. 23) in diesem Sinne als Effekt von Politik qua Unterbrechung von Polizei zu verstehen: Es entsteht erst aus dem Dissens zwischen Zählung und Ungezählten und verweist damit insofern auf einen Horizont radikaler Gleichheit, als im Moment der Aufhebung der Zählung die Gleich-Gültigkeit aller sprechenden Subjekte aufgezeigt wird. Die Demokratie wiederum stellt „die Einsetzung selbst der Politik“ (Rancière 2008, S. 19) dar, die so gesehen nur eine Politik der Gleichheit sein kann und damit auf einen ,demokratische[n]“ bzw. „emanzipatorische[n] Apriorismus“ (Marchart 2010, S. 178) im Politikverständnis Rancières verweist.

Dass Rancière vom Volk und nicht von der Macht als originärer Leerstelle ausgeht, hat eine Reihe von Implikationen: Zunächst wird , das Volk' quasi als das Reale der polizeilichen Verkörperung des Sinnlichen verstanden, dessen Einsetzung bzw. „politische Subjektivierung“ wiederum mit der Logik von Verkörperung bzw. Zählung als solcher bricht, anstatt wie bei Lefort auf die potenzielle Gefahr von deren Verabsolutierung hinzudeuten. Insofern bildet das Rancière'sche Pendant zum totalitären Imaginären einer Gesellschaft bzw. eines Volkes ohne Teilung bei Lefort die ,post-demokratische“ Vorstellung einer Demokratie ohne Dissens und damit ohne Volk: In beiden Fällen handelt es sich um den totalisierenden und zutiefst demokratiefeindlichen Anspruch, das Reale vollständig zu meistern, die Kontingenz außer Kraft zu setzen, „die Gemeinschaft in sich selbst einzuschließen“ (Rancière 2002, S. 131) - und damit letztlich ,ohne Volk [zu] regieren, d.h. ohne die Teilung des Volks regieren; ohne Politik regieren“ (Rancière 2012, S. 96). Auch wenn Lefort und Rancière damit letztlich auf die gleiche Gefahr hinweisen - nämlich die Verdeckung der Teilung und der Kontingenz -, besteht für Rancière die Gefahrenquelle nicht (nur) in der totalitären Verabsolutierung, sondern insbesondere in der postdemokratischen Verdrängung des ,Volkes‘. Einem autoritären bzw. totalitären Populismus, der sich im Namen eines, Volkes “ über den demokratischen Dissens stellt, und einer technokratischen Verschweigung der Volkssouveränität im Namen der ,Alternativlosigkeit“ ist nämlich eine polizeiliche Logik gemeinsam, die das ,Volk' als Kategorie der Politik stilllegen will. Beispielhaft für diese Betrachtungsweise ist Rancières (2017) Kritik an der Verwendung des Populismusbegriffs im öffentlichen Diskurs in Frankreich: Rassistische Praktiken in staatlichen Institutionen sind etwa nicht das Produkt rechtspopulistischer Mobilisierung, so Rancière, sondern Bestandteil der polizeilichen Machttechniken des Staates, die durch die Berufung auf die vermeintliche Gefahr des ,Populismus“ sowie die angebliche Empfänglichkeit des ,Volkes“ für rassistische Forderungen erst legitimiert werden - und dadurch die Kategorie des ,Volkes“ mit dem Bild gefährlicher und ressentimentgeladener Massen zu fixieren versuchen, um die politische Einsetzbarkeit des Begriffs zu neutralisieren. 
Rancières Problematisierung der postdemokratischen, und nicht nur der totalitären, Gefährdung der radikaldemokratischen Kontingenz - eine Tendenz, die sich in Mouffes (2017) Kritik der „Postpolitik“ wiederfindet - lässt den in der liberalen Lefort-Rezeption gängigen Dualismus zwischen institutionalisierter Demokratie und demokratiefeindlichem Populismus umso fragwürdiger erscheinen: Gerade wenn die institutionelle Konstellation selbst postdemokratisch und kontingenzgefährdend wirkt, liegt es nahe, dass durch das populistische Reklamieren eines abwesenden ,Volkes‘ gegen die Ordnung der Polizei Momente der Politik entstehen können. Dabei stellt der Populismus die Rancière'sche (radikale) Demokratie vor ein anderes Problem: Denn auch ein demokratischer Populismus, der „das Volk“ als Figur der Anteillosen gegen die herrschende Ordnung wendet - ohne daraus einen totalisierenden Herrschaftsanspruch zu ziehen -, kann sich kaum auf egalitär-politische Momente beschränken und auf eigene (polizeiliche) Herrschaftsansprüche verzichten. Auch das Beispiel der Platzbewegungen legt nahe, dass radikal-egalitär anmutende Protestbewegungen nicht nur herrschende Ordnungen des Sinnlichen stören, sondern durchaus auch alternative Herrschaftsansprüche formulieren können, wie Gerbaudo (2017b) mit seiner Analyse des populistischen und antioligarchischen $\mathrm{Ci}$ tizenship-Verständnisses bei 15-M und Aganaktismenoi aufgezeigt hat. Hierin zeigt sich in exemplarischer Manier die Unmöglichkeit, eine ,reine“ Politik nach Rancière zu betreiben (vgl. Chambers 2011), sofern die Politik als flüchtiges, ereignishaftes Moment und das Begriffspaar Politik/Polizei als zwei konstitutive „Logiken des menschlichen Zusammenseins“ (Rancière 2002, S. 39) aufgefasst wird. Ebenfalls am Beispiel von 15-M und Aganaktismenoi zeigen Prentoulis und Thomassen (2013, S. 181) auf, dass das Rancière'sche Moment der Politik-als-Gleichheit wiederum die Schaffung neuer - auch vertikaler - Repräsentationsstrukturen im Rahmen von Protestbewegungen nach sich ziehen kann, damit Prinzipien wie Horizontalität und Gleichheit überhaupt zur Geltung kommen: „There is no horizontality without verticality, and no equality without inequality, because horizontality and equality are not natural, but must be instituted."

In der schieren Unmöglichkeit, Populismus oder gar jegliche Form von Politik zu betreiben, ohne irgendwann in Polizei überzugehen, drückt sich das ambivalente Dazwischen-Sein des Populismus aus, der einerseits ein ,Volk' gegen die herrschenden Machtverhältnisse reklamiert und damit eine prototypische Erscheinungsform von Politik qua Unterbrechung von Polizei darstellt, aber andererseits auch einen Repräsentationsanspruch par excellence - und damit die Überführung von Politik in eine neue arche - in sich trägt. An dieser Stelle argumentiert Agridopoulos (2018, S. 244 f.) in Bezug auf die Figur der Anteillosen, dass Rancières Denken nichts weniger, aber auch nichts mehr als eine ,linkspopulistische Facette“ aufweist, sofern zwar die antagonistische Gegenüberstellung von anteillosem Volkssubjekt und Regime der Polizei vorhanden ist, aber andererseits ,,auch keine Aspiration des Anteils der Anteillosen eine populäre Demokratie als Gegenhegemonie [...] innerhalb eines Staates zu etablieren“. Im selben Zuge lässt sich konstatieren, dass die Politik nach Rancière auch nur eine Facette des Populismus bildet, und zwar neben der der Polizei, die gerade für einen hegemonietheoretisch begründeten Populismusbegriff - der wiederum bei Laclau und Mouffe in Erscheinung tritt - ebenso wenig wegzudenken ist. 


\section{Von der radikalen Demokratietheorie Laclaus und Mouffes zur Populismustheorie Laclaus}

\subsection{Radikale Demokratie im Frühwerk Laclaus und Mouffes}

Mit dem gemeinsamen Frühwerk Ernesto Laclaus und Chantal Mouffes avanciert die radikale Demokratietheorie nicht nur zu einer weiteren postfundamentalistischen Spielart des radikalen Kontingenzgedankens, sondern auch zu einer Hegemonietheorie der Politik, die die Autonomie ,demokratischer Kämpfe“ als weitere konstitutive Dimension radikaler Demokratie in den Vordergrund stellt. In Hegemonie und radikale Demokratie unternehmen Laclau und Mouffe zunächst eine Dekonstruktion des Marxismus, die aufzeigt, dass das Beharren auf einem aus der ökonomischen Klassenposition abgeleiteten einheitlichen Klassensubjekt im Laufe der Geschichte der Zweiten und Dritten Internationale an Grenzen stößt und dass Gramscis Konzept der Hegemonie wiederum ein Terrain öffnet, wo sich „Gesellschaft“ als Ergebnis kontingenter diskursiver Fixierungen und nicht als objektiv gegebene Totalität theoretisieren lässt. Für Laclau und Mouffe (2012, S. 149) wird soziale Realität grundsätzlich durch die differenz- und äquivalenzlogische In-Bezug-Setzung diskursiver Elemente zueinander konstituiert, wobei die Identitäten der dadurch konstruierten diskursiven Elemente eine letztliche „Nicht-Fixiertheit“, d.h. Kontingenz, aufweisen. So verstanden bildet „Gesellschaft“ immer ein konstitutiv unvollständiges Objekt, das als (partielles) System von Differenzen erst durch eine antagonistische Spaltung bebzw. gegründet werden kann, die den verschiedenen differenziellen Positionen nach der Logik der Äquivalenz eine kollektive Identität gegen ein konstitutives Außen verleiht (z.B. alle ,Bürger' gegen ,Adel', alle ,Arbeiter' gegen ,Kapital', die gesamte ,Nation' gegen eine, fremde Macht'). Hegemonie bezeichnet also den Kampf um partielle Fixierungen des Sozialen um Knotenpunkte (z.B. ,Bürger‘, ,Nation`), wobei das Politische qua Antagonismus als Moment der „Einrichtung des Sozialen“ einerseits und die Sedimentierung bzw. Verstetigung politisch instituierter Sinnfixierungen andererseits stets im Wechselspiel miteinander stehen (Laclau und Mouffe 2012, S. 193; vgl. auch Laclau 1990).

Damit besteht für Laclau und Mouffe (2012, S. 192) eine konstitutive Dimension ihres radikalen Demokratieverständnisses in der „Anerkennung der Pluralität und Unbestimmtheit des Sozialen“, sprich: Antagonismen lassen sich grundsätzlich nicht auf apriorisch ,privilegierte[] Bruchpunkte[]“ (wie etwa ,die Arbeiterklasse“) zurückführen und können durch ganz verschiedene emanzipatorische Kämpfe zustande kommen. An dieser Stelle machen die Autor*innen einen entscheidenden Sprung, indem sie sich auf die Lefort'sche Idee der Demokratie als ,neue[r] Art und Weise der Instituierung des Sozialen“ berufen und für eine Radikalisierung der Demokratie plädieren, die die im symbolischen Dispositiv der Demokratie angelegten Freiheits- und Gleichheitsrechte für immer mehr Subjekte einfordert und damit eine Äquivalenzkette unterschiedlicher ,demokratischer Kämpfe“ gegen Unterdrückungsverhältnisse aller Art formiert (Laclau und Mouffe 2012, S. 196). Damit zielt das radikaldemokratische Projekt auf ,,die Vervielfachung von Antagonismen und die Konstruktion einer Pluralität von Räumen“ ab (Laclau und Mouffe 2012, S. 237), wobei jeder „,demokratische Kampf“ innerhalb des radikaldemokratischen 
hegemonialen Blocks als autonomer Ort eines eigenständigen Antagonismus aufgefasst wird, indem etwa feministische und antirassistische Kämpfe sich erst in antagonistischer Abgrenzung gegen patriarchale bzw. rassistische Herrschaftsverhältnisse (be)gründen. Folglich bildet ,die Autonomie sozialer Bewegungen eine Voraussetzung für das Auftauchen von Antagonismen als solchen“ und damit eine konstitutive Dimension radikaler Demokratie (Laclau und Mouffe 2012, S. 173). Radikale Demokratie nach Laclau und Mouffe (2012, S. 227f.) lässt sich insofern als Ineinandergreifen von Kontingenz und Autonomie auffassen, sofern die Akzeptanz der irreduziblen Pluralität des Sozialen (Kontingenz) in die Anerkennung der ebenso irreduziblen Pluralität der Kämpfe um Demokratie (Autonomie) übergeht, weshalb ein spezifisch radikaldemokratisches Hegemonieprojekt sich das „,Prinzip der demokratischen Äquivalenz"“ aneignen und damit die formale Logik der Äquivalenz „durch die Logik der Autonomie ergänz[en]/begrenz[en]“ muss.

Für den Populismus hat das Frühwerk Laclaus und Mouffes bereits die Implikation, dass ein populistischer Diskurs (nur) in dem Maße radikaldemokratisch sein kann, wie er die Autonomie der äquivalenzlogisch verlinkten „demokratischen Kämpfe“" anerkennt und fördert. Als eine Art Gegenpol zu radikaler Demokratie führen Laclau und Mouffe (2012, S. 210ff.) das Beispiel des neokonservativen „Rechtspopulismus“ an, der - ähnlich wie bei Stuart Halls (1988) Analyse des Thatcherismus als ,authoritarian populism“ - einen Anti-Establishment-Diskurs mit einem antiegalitären Freiheitsverständnis verknüpft und damit das demokratische Terrain von Freiheit und Gleichheit untergräbt. Allerdings wäre auch ein Populismus, der „Freiheit und Gleichheit für alle“ auf die Fahnen schreibt, dann problematisch, wenn die Freiheits- und Gleichheitsforderungen innerhalb der Äquivalenzkette nicht als eigenständige Kämpfe artikuliert, sondern auf den einen vermeintlich zentralen Kampf zurückgeführt werden - wenn z.B. ,Frauenrechte' nicht etwa zunächst gegen ,Patriarchat' konstituiert, sondern auf ein Moment der Frontstellung, Volk' gegen ,Oligarchie“ reduziert werden. Es gibt also eine grundlegende Spannung zwischen Autonomie und hegemonialer Führung, die Laclau und Mouffe wiederum durch das Ineinandergreifen von Autonomie und Äquivalenz in „,demokratische Äquivalenz“ radikaldemokratisch einzurahmen versuchen; in anderen Typen von Hegemonie, wo die Autonomie der Kämpfe nicht als konstitutiv anerkannt wird, besteht hingegen durchaus die Gefahr, dass ein zentraler hegemonialer Führungsanspruch die Autonomie der sonstigen Positionen innerhalb der Kette untergräbt. In diesem Sinne argumentieren Laclau und Mouffe (2012, S. 183) sogar, dass „Autonomie selbst nur in Form eines umfassenderen hegemonialen Kampfes verteidigt und entwickelt werden kann“, der den Stellenwert von Autonomie überhaupt mitdenkt.

\subsection{Laclaus Theorie des Populismus}

In der späteren Populismustheorie Laclaus verschiebt sich allerdings das konzeptuelle Terrain dahingehend, dass Autonomie keine konstitutive Rolle mehr spielt und dass die Logik der Repräsentation - und damit die vertikale Dimension hegemonialer (Zusammen-)Führung - stattdessen in den Vordergrund rückt (vgl. Kim 2019). Eine erste wichtige Verschiebung besteht darin, dass Laclau die Kategorie der demand (,Forderung“) als ,elementary unit of politics“ (Marchart 2018, S. 111) 
betrachtet und davon ausgehend seinen Begriff des Populismus entwickelt. Jede Forderung ruft grundsätzlich eine Machtinstanz zu ihrer Erfüllung auf; dabei wird ein antagonistischer Bruch mit der Machtinstanz nicht durch ,democratic demands“ zustande gebracht, die differenzlogisch in ihrer Partikularität artikuliert werden und damit das bestehende Terrain als solches nicht infrage stellen, sondern erst durch deren äquivalenzlogische Artikulation als miteinander verlinkte ,popular demands“, die durch die negative Dimension der Nicht-Erfüllung von der Machtinstanz eine Äquivalenzkette bilden und damit eine antagonistische Grenze gegen diese erzeugen. Kennzeichnend für den Populismus ist, dass ,das Volk' als Kollektivsubjekt der unerfüllten Forderungen einem Machtblock antagonistisch gegenübergestellt und damit als Knotenpunkt konstruiert wird, der unterschiedliche Forderungen gegen den Machtblock verbindet. Allerdings geht Laclau (2005a, S. 162) noch einen Schritt weiter und argumentiert, dass der Name eines Volkssubjekts im Populismus nicht nur die partielle Fixierungsfunktion eines Knotenpunkts einnimmt, sondern auch als leerer Signifikant die Identität der äquivalenzlogischen Einheit repräsentiert und damit erst konstituiert, indem er tendenziell auf eine eigene differenzielle Partikularität verzichtet, um der Äquivalenzkette den Namen einer abwesenden positiven Einheit $\mathrm{zu}$ verleihen. Es handelt sich also um eine Repräsentationsfunktion par excellence, da ,Volk' weiterhin einen partikularen Signifikanten darstellt, der sich allerdings einer partikularen Differenz (tendenziell) entleert, um für das Ganze zu stehen. Dabei kommt dieses Ganze aber auch erst durch den Akt der Repräsentation zustande, indem die Identität der unterschiedlichen Forderungen als populare Identität erst mit der Benennung als ,Volk“ konstituiert wird: ,,populist discourse does not simply express some kind of original popular identity; it actually constitutes the latter" (Laclau 2005b, S. 48). Diese Repräsentationslogik ist für jede Art von kollektiver Identitätsbildung konstitutiv - und tritt beim Populismus eben prototypisch zu Tage: ,relations of representation are, on the contrary, the primary terrain within which the social is constituted" (Laclau 2005b, S. $48 \mathrm{f}$.).

Im Gegensatz also zu ,the increasing centrality that I have given, in my recent work, to the logic of representation“ (Laclau 2015, S. 258) ist der Begriff der Autonomie in Laclaus Populismustheorie nicht mehr konstitutiv dabei: Denn die Grundeinheiten der Äquivalenzkette sind nicht mehr ,demokratische Kämpfe“, die sich zuerst als Orte eigener Antagonismen konstituieren, sondern ,demokratische Forderungen“, die auf sich alleine gestellt kein antagonistisches Terrain (be)gründen können und hierzu im Gegenteil repräsentative Mechanismen brauchen. In diesem Zusammenhang wird sogar dem leeren Signifikanten als repräsentativer Instanz eine gewisse Autonomie zugeschrieben: Laclau (2005a, S. 93) spricht nämlich von ,the construction of the ,people" as a crystallization of a chain of equivalences in which the crystallizing instance has, in its autonomy, as much weight as the infrastructural chain of demands which made its emergence possible“. Damit wird die vertikale Dimension hegemonialer Führung eines repräsentativen Signifikanten als ebenso konstitutiv für den Populismus betrachtet wie die horizontale Äquivalenzverkettung, die wiederum aber keine Autonomie (im Sinne der Konstituierung eines eigenen antagonistischen Terrains) der verketteten Positionen mehr voraussetzt. An dieser Stelle folgt der vielleicht kontroverseste Schritt Laclaus, diese vertikale Repräsentationsfunktion vom Namen eines Volkssubjekts in jenen einer Führungsfigur zu 
überführen: Denn durch die äquivalenzlogische Zusammenführung wird eine Singularität konstruiert, aber ,the extreme form of singularity is an individuality“ - mit dem Ergebnis, dass ,,singularity [leads] to identification of the unity of the group with the name of the leader" (Laclau 2005a, S. 100). Der Name einer Führungsfigur tritt also als eine Art leerer Signifikant in letzter Instanz in Erscheinung als letztverfügbarer Kristallisationspunkt für populistische Identifikation -, der die verbindende Funktion von ,Volk' ergänzt, insbesondere in dem Maße, wie diese bei zunehmender Ausdehnung der Äquivalenzkette verloren geht. Dabei liegt nahe, dass diese Ergänzungsfunktion theoretisch auch von anderen Knotenpunkten - wie z.B. ,Demokratie ‘ - erfüllt werden könnte; konstitutiv für den Populismus ist allerdings, dass die Repräsentationslogik vom Namen eines Volkssubjekts in irgendeinem (unbestimmten) Maße in den Namen einer Führungsfigur übergeht.

Aus den Verschiebungen von der radikalen Demokratietheorie Laclaus und Mouffes zur Populismustheorie Laclaus ergibt sich eine grundlegende Spannung, die sich wiederum mit dem übergreifenden konzeptuellen Vokabular der Diskurs- und Hegemonietheorie konkretisieren lässt: Während radikale Demokratie bei der Konstruktion von Hegemonie die horizontale Dimension der Autonomie ,demokratischer Kämpfe“ als konstitutiv hervorhebt, verlagert sich beim Populismus die konstitutive Dimension auf die vertikale Repräsentations- und Identifikationsfunktion von Volkssubjekt und Führungsfigur. Auch wenn beide Dimensionen von horizontaler Äquivalenzverkettung und hegemonialer Führung sowohl in der radikalen Demokratie- als auch in der Populismustheorie vorhanden sind, wird die horizontale Dimension der Äquivalenz im Spätwerk nicht mehr in Bezug auf Autonomie gedacht. Während für radikale Demokratie sogar ein besonderer Typus von Äquivalenz gilt - , ,demokratische Äquivalenz“, die der Autonomie der verketteten Positionen Rechnung trägt -, wird beim Populismus eine besonders ausgereifte Form vertikaler Repräsentation als konstitutives Merkmal vorausgesetzt und die Verknüpfung von Autonomie und Äquivalenz hingegen tendenziell fallen gelassen. Sofern aber diese Repräsentationslogik im Zusammenspiel mit Äquivalenzverkettung und antagonistischer Grenzziehung für jede kollektive Identitätsbildung konstitutiv ist, weist der Populismus einen exemplarischen Charakter für das Politische als solches auf - und wird sogar „synonymous“ mit diesem im Sinne eines ,moment of institution of the social“" (Laclau 2005a, S. 154). Dies heißt wiederum, dass der Populismus auch ein kaum verzichtbares Moment radikaler Demokratie darstellen muss: In der Tat argumentiert Laclau (2005c, S. 259) in einem kleineren Aufsatz, ,radical democracy is always ,populist““ insofern, als die Radikalisierung der Demokratie ,presupposes the constitution of an underdog as a political actor“. Im selben Zuge argumentiert er aber auch, dass es mindestens zwei weitere konstitutive Dimensionen der Radikalisierung der Demokratie gibt: erstens die institutionalisierte liberale Demokratie und deren Versprechen „Freiheit und Gleichheit für alle“, das es auf immer mehr Subjekte auszuweiten gilt; zweitens die Pluralität unterschiedlicher ,democratic demands“" und deren Partikularitätsansprüche. Die beiden Dimensionen ähneln durchaus dem früheren ,,radikalen und pluralen“ Demokratieverständnis Laclaus und Mouffes, werden hier aber neben dem Populismus als drei konstitutive, in letzter Instanz miteinander unvereinbare Momente radikaler Demokratie aufgefasst: Alle drei sind nötig, aber ,the unilateralisation of any of the three meanings leads, in 
actual fact, to the collapse of democracy“ (Laclau 2005c, S. 261). Radikale Demokratie wird damit zum Ort eines unauflösbaren Spannungsverhältnisses zwischen ihren konstitutiven Momenten, zu denen auch das populistische gehört.

Damit lässt sich in Laclaus eher wenig bekanntem Aufsatz die gesamte Spannungslinie zwischen Populismus und radikaler Demokratie in verdichteter und zugespitzter Form auffinden: Populismus bildet sowohl ein konstitutives Moment als auch eine Grenze radikaler Demokratie, sofern die antagonistische Konstruktion eines Volkssubjekts gegen die herrschenden Machtverhältnisse zwar eine notwendige Dimension der Radikalisierung der Demokratie darstellt, aber mit deren anderen Dimensionen wie etwa dem Pluralismus - oder, mit der früheren Theorie Laclaus und Mouffes gedacht, der Autonomie demokratischer Kämpfe - in einem unauflösbaren Spannungsverhältnis steht. Was im Spätwerk Laclaus an radikaldemokratischem Gehalt übrig bleibt, ist also die postfundamentalistische Anerkennung der Kontingenz, die aber nicht mehr in die radikal-pluralistische Dimension der Autonomie der Kämpfe übergehen muss; Nonhoff (2019, S. 344) argumentiert in diesem Zusammenhang, dass

der Kern von Laclaus radikaldemokratischem Denken sich weder primär auf radikal-pluralistische Kämpfe bezieht noch radikale Demokratie mit Populismus in eins setzt. Entscheidend ist vielmehr die im Begriff des Antagonismus und im unaufhebbaren Zusammenspiel von Äquivalenz und Differenz zum Ausdruck kommende Grundlosigkeit des Sozialen.

Wenn die radikale Demokratie damit weder im Populismusbegriff als konstitutive Dimension angelegt noch auf diesen reduziert wird, öffnen sich immerhin Spielräume für eine produktive, wenn auch notwendig kontingente und spannungsreiche, Koexistenz von Populismus und radikaler Demokratie. Denn ein populistischer Diskurs muss nicht radikaldemokratisch sein, kann dies aber in dem Maße werden, wie er den äquivalenzlogisch verketteten Positionen eine autonome Stellung einräumt - wenn etwa ,Frauenrechte“ gegen spezifisch patriarchale Verhältnisse artikuliert und nicht nur entlang einer zentralen Konfliktlinie (z.B. gegen ,die Oligarchie‘) der Seite des Volkssubjekts zugeordnet werden. Die Herausforderung für einen radikaldemokratischen Populismus würde darin bestehen, „demokratische Forderungen“ zusätzlich als autonom konstituierte ,demokratische Kämpfe“ anzuerkennen, die mit Laclau und Mouffe eine „Pluralität von Räumen“ und damit auch eine gewisse organisatorische Autonomie beanspruchen können, während der Name von Volkssubjekt und Führungsfigur andererseits eine zentrale verbindende Funktion im Diskurs einnimmt. An dieser Stelle deutet sich bereits an, dass Fragen der Organisation eine wichtige Rolle dabei spielen, wie das Verhältnis von Populismus und radikaler Demokratie im Rahmen von Parteien und Bewegungen ausgehandelt wird - ein Faden, der in den jüngsten Interventionen Mouffes verstärkt aufgegriffen wird.

\subsection{Mouffes Plädoyer für den Linkspopulismus}

Mouffes streitbares Eintreten für den Linkspopulismus beruht auf der Überzeugung, dass eine radikaldemokratische Dimension des Linkspopulismus nicht nur möglich, sondern auch nötig ist und umgekehrt - dass sich ein radikaldemokratisches Pro- 
jekt den Linkspopulismus aneignen muss -, damit ein linkes Hegemonieprojekt im neoliberalen Zeitalter Aussichten auf Erfolg haben kann. Mouffe diagnostiziert eine Situation, in der die Hegemonie des Neoliberalismus im westeuropäischen Kontext zu zunehmenden Oligarchisierungstendenzen sowie einer ,postpolitischen“ Verwässerung von Links-Rechts-Gegensätzen geführt habe:

Lange Zeit dachte ich: Um gegen die postpolitische Tendenz zu kämpfen und die Bedingungen für eine agonistische Konfrontation zu schaffen, die die Radikalisierung der Demokratie ermöglicht, ist es notwendig, der Frontstellung links/rechts eine neue Kraft zu verleihen. Aber mir wurde bewusst, dass dies unter den aktuellen Bedingungen nicht der adäquateste Weg war und dass es notwendig sein wird, die Front auf andere Art und Weise zu etablieren, und zwar über die Konstruktion eines transversalen und progressiven Kollektivwillens, der die vom Neoliberalismus produzierten neuen oligarchischen Formen herausfordern könnte. Das ist es, was ich „Linkspopulismus“ nenne. (Mouffe in Errejón und Mouffe 2015, S. 112)

Mouffes Präferenz für den Linkspopulismus ist also strategisch-zeitdiagnostischer Natur und als Antwort auf eine „Postpolitik“ gedacht, die das Politische von der Politik verdränge und insbesondere die ,demokratische Tradition“ der konflikthaften Auseinandersetzung um die Volkssouveränität untergrabe (Mouffe 2017). In Anlehnung an Laclau versteht Mouffe (2018, S. 20f.) Populismus ,als eine Diskursstrategie, die [...] zu einer Mobilisierung der ,Benachteiligten“ gegen, die an der Macht" aufruft" und im Fall des Linkspopulismus ,das Volk' gegen ,die Oligarchie " mit dem Ziel einer „Wiederherstellung und Vertiefung der Demokratie“ reklamiert (Mouffe 2018, S. 16). Für Mouffe (2018, S. 30) muss der Linkspopulismus damit die Impulse „horizontalistische[r] Bewegungen“ einerseits aufgreifen, die auf öffentlichen Plätzen ,echte Demokratie jetzt“ lautstark eingefordert haben, diese aber auch in die Hegemoniekämpfe innerhalb der Institutionen hineintragen. Das Projekt einer Radikalisierung der Demokratie braucht also den Linkspopulismus: Denn radikaldemokratischer Protest muss auch den Kampf innerhalb der institutionell geregelten Politik und insbesondere um die Konstruktion eines ,Volkes ' annehmen - die wiederum im ,populistischen Moment“ durch den Aufstieg des Rechtspopulismus längst in den Vordergrund gerückt ist -, um transformatorische Wirkung entfalten zu können.

In welcher Form soll aber umgekehrt die Radikaldemokratisierung des Linkspopulismus erfolgen? Für Mouffe (2018, S. 53) gilt es einerseits, ,die linkspopulistische Strategie in der demokratischen Tradition zu verankern" und das im symbolischen Dispositiv der Demokratie angelegte Freiheits- und Gleichheitsversprechen für immer mehr Subjekte einzufordern. Damit fehlt allerdings - wie im Spätwerk Laclaus - die Autonomie demokratischer Kämpfe im konzeptuellen Vokabular der Populismustheorie; andererseits deutet Mouffe im 2015 erschienenen Gesprächsband mit dem damaligen Podemos-Chefstrategen Íñigo Errejón auf ebendiesen Aspekt hin, wenn sie vom Linkspopulismus als ,neue[r] politische[r] Organisationsform“ spricht, die den vielfältigen ,demokratischen Forderungen, die in unseren Gesellschaften existieren [...] horizontale Ausdrucksformen“"verleihen und damit auch über „die vertikalistische Form der Partei“ hinausgehen müsse (Errejón 
und Mouffe 2015, S. 113). Hier kommt die konstitutive Spannung zwischen Populismus und radikaler Demokratie, zwischen horizontaler Autonomie und vertikaler Repräsentation abermals zum Ausdruck, wird dabei gleichzeitig auf die Organisationsebene als Frage der Aushandlung beider Logiken verlagert und lokalisiert: Es geht letztlich um die Erfindung einer neuen Organisationsform, die sowohl die Autonomie demokratischer Forderungen als auch deren vertikale Zusammenführung um ein Volkssubjekt mitdenkt.

\section{Ein Blick auf die Empirie: Zwischen Bewegungsparteien und Volksparteien neuen Typs}

Es öffnet sich also ein weiter Blick auf das Verhältnis von Populismus und radikaler Demokratie, für dessen Erforschung die sog. Platzbewegungen sowie die diversen Versuche von Parteiformationen, an die Protestdiskurse der Plätze anzuknüpfen, ein besonders aufschlussreiches Feld bieten. Aus der theoretischen Analyse geht zunächst hervor, dass sich die verschiedenen Spielarten radikaler Demokratietheorie allesamt durch Denkfiguren der radikalen Kontingenz auszeichnen; dass dabei die radikale Demokratietheorie Laclaus und Mouffes die Dimension der Autonomie demokratischer Kämpfe einführt; und dass die spätere Populismustheorie Laclaus eine klare Akzentverschiebung von Autonomie zu Repräsentation nach sich zieht und damit der grundlegenden Spannung zwischen Populismus und radikaler Demokratie konzeptuell zugespitzten Ausdruck verleiht, was Mouffe wiederum insbesondere als Frage der Organisation deutet. In empirisch-analytischer Hinsicht stellt sich also die Frage, wie das Verhältnis von Populismus und radikaler Demokratie, zwischen Autonomie und Repräsentation durch politische Praktiken ausgehandelt wird - vor dem theoretischen Hintergrund, dass Prinzipien wie etwa „Autonomie“ oder „Horizontalität“ erst durch Strukturen instituiert werden und dabei auch repräsentative bzw. vertikale Gestalt annehmen können (vgl. Prentoulis und Thomassen 2013), weshalb dieses Spannungsverhältnis einerseits eben unauflösbar, andererseits aber auch produktiv ist, indem es durchaus Spielräume für eine gegenseitige Befruchtung zulässt. Grundsätzlich lässt sich konstatieren, dass Parteien und Bewegungen bei der organisatorischen Aushandlung der beiden Logiken mit einem Kontinuum an Möglichkeiten konfrontiert sind: Sie können tendenziell entweder auf die horizontale Integration autonom organisierter Bewegungen einerseits oder auf die vertikale plebiszitäre Bindung zwischen einer Führung und einem möglichst breit partizipierenden, Volk' andererseits setzen.

Diese Formulierung ähnelt einerseits der theoretisch nachgezeichneten Spannung zwischen Autonomie und Repräsentation, andererseits aber auch diversen Annäherungen an den Begriff der „Bewegungspartei“ innerhalb der Parteien- und Bewegungsforschung. In dieser Literatur geht es um die Frage, wie sich Protestbewegungen institutionalisieren und wie sich neue Formen der Parteiorganisation als Folgephänomene von Protestmobilisierungen herausbilden, was zunächst insbesondere in Bezug auf Grüne bzw. „linkslibertäre“ Parteien erforscht wurde (vgl. etwa Kitschelt 1986; Richardson und Rootes 1994; Mayer und Ely 1998). In einem der wenigen konzeptuellen Systematisierungsversuche definiert Kitschelt (2006, S. 280 f.) Bewe- 
gungsparteien in Bezug auf einen niedrigen Formalisierungsgrad der Organisationsstruktur sowie die Verknüpfung von ,,activities within the arenas of formal democratic competition with extra-institutional mobilization“; das erste Definitionskriterium bedeutet wiederum, dass es bei der Organisationsform einer Bewegungspartei ein Möglichkeitskontinuum gibt: von der Zentrierung um ,a charismatic leader with a patrimonial staff and personal following“ einerseits bis hin $\mathrm{zu}$,grassroots democratic, participatory coordination among activists“" andererseits. Ähnlich argumentieren Della Porta et al. (2017, S. 23) in ihrer Untersuchung von ,movement parties against austerity“, dass diese sich in der Organisationsfrage zwischen „decentralized organization“ und ,trends towards personalization“ bewegen. Aus einer diskurs- und hegemonietheoretischen Perspektive in Anlehnung an Laclau und Mouffe handelt es sich wieder um die beiden Pole von Autonomie und Repräsentation, die in der radikalen Demokratie bzw. im Populismus prototypisch zutage treten.

Damit öffnet sich die Möglichkeit, unterschiedliche Spielarten der organisatorischen Aushandlung von Populismus und radikaler Demokratie konzeptuell einzuordnen. Konkret lässt sich eine Unterscheidung zwischen Bewegungsparteien und Volksparteien neuen Typs treffen: Erstere orientieren sich primär an der horizontalen Integration autonom organisierter Bewegungen, während Letztere ein möglichst breit partizipierendes, Volk' als Repräsentationssubjekt interpellieren und auf dessen vertikale plebiszitäre Bindung zu einer Führung setzen. Bewegungsparteien in diesem engeren Sinne sind empirisch nicht häufig auffindbar: Neben der CUP in Katalonien wäre ein aus theoretischer Perspektive prototypisches, wenn auch im Parteiensystem äußerst marginales Beispiel das ukrainische Linkspartei-Projekt „Soziale Bewegung“ (SR), das die Parteiform als ,kollektives Instrument“ horizontal verlinkter Bewegungsakteure versteht und insbesondere auf die Einbindung von Vertreter*innen unabhängiger Gewerkschaften ins Organisationskomitee setzt (vgl. Kim 2019). Damit wird aber eine radikaldemokratische Politik der Autonomie in eine Repräsentationslogik überführt, was wiederum auf die unauflösbare Spannung horizontal/vertikal verweist. Darüber hinaus setzt die SR einen linkspopulistischen Diskurs von „Volksmacht statt absoluter Macht der Oligarchen“ ein, der allerdings die Repräsentationsfunktion von ,Volk“ tendenziell nicht in die Zentralität einer Führungsfigur überführt und damit eher zum Pol des ,leaderless populism“ (Gerbaudo 2017a) tendiert. Volksparteien neuen Typs hingegen - wie z. B. Podemos und France Insoumise - verknüpfen einen linkspopulistischen Diskurs mit einer Repräsentationslogik, die vom Namen eines Volkssubjekts in die verbindende Funktion einer Führungsfigur im Diskurs übergeht und etwa durch den Einsatz digitaler Instrumente (z.B. Online-Referenden; vgl. Gerbaudo 2018) sowie den Abbau traditioneller Partizipationshürden (z.B. Mitgliederbeiträge) ein möglichst breit partizipierendes ,Volk' auch innerhalb der Parteiorganisation als Repräsentationssubjekt aufruft und in ein vertikales plebiszitäres Verhältnis zur Führung setzt (vgl. Kioupkiolis 2016; Rendueles und Sola 2018; Kim 2019). Es handelt sich also um den konzeptuellen Gegenpol zu Bewegungsparteien einerseits, die organisierte Bewegungsakteure als innerparteiliches Repräsentationssubjekt interpellieren (und diese etwa mit Plattformen innerhalb der Partei ausstatten), und zu „Mitgliederparteien“ andererseits (vgl. Wiesendahl et al. 2009), die eine beitragszahlende und organisatorisch klar abgrenzbare Mitgliederbasis als Repräsentationssubjekt im Innern aufrufen. Auch 
wenn Parteien, die den letzten beiden Typen zugeordnet werden können, durchaus auch populistisch an das, Volk' gegen eine Machtelite appellieren können, besteht ein wichtiger Unterschied darin, inwiefern diese Anrufung eines Volkssubjekts performativ in die eigene Organisationslogik hineingetragen wird und damit auch die herkömmliche Trennung zwischen der Mitgliedschaft und deren Außen destabilisiert wird.

Dabei bestehen auch innerhalb der Volksparteien neuen Typs durchaus Spielräume für radikaldemokratische Momente der Autonomie (Mouffes ,horizontale Ausdrucksformen“): im Fall von Podemos etwa durch autonome Bündnisse auf kommunaler Ebene, die von der Partei zwar unterstützt werden, organisatorisch aber ein getrenntes Leben führen und jeweils einer Logik der lokalen Bewegungsverankerung und Äquivalenzverkettung folgen (in Barcelona z. B. mit Ada Colau aus der Bewegung gegen Zwangsräumungen). Das Beispiel Podemos verweist außerdem auf die Besonderheiten eines ,reflexive populism“ (Kioupkiolis 2016), der sich explizit an der Theorie Laclaus orientiert (vgl. etwa Iglesias 2015 zur „Hypothese Podemos“) und sich dabei auch zur Autonomie demokratischer Kämpfe bekennt weshalb weder Podemos noch irgendeine Kraft „die Partei von 15-M“ sein könne (vgl. Errejón in Errejón und Mouffe 2015) und soziale Bewegungen wie 15-M gar „unrepräsentierbar“ seien (Iglesias auf der öffentlichen Vorstellung von Podemos im Januar 2014) -, daraus aber wiederum stark vertikale Schlussfolgerungen zieht: Denn gerade vor dem Hintergrund, dass eine Bewegungspartei-Strategie des Parteiaufbaus um organisierte Bewegungen nicht infrage kam, ließ sich die Strategie leicht rechtfertigen, von vornherein auf die vertikale Identifikation mit einer ,,medialen Führung“" zu setzen (vgl. Iglesias 2015). Die Nebeneinanderstellung etwa von SR als Bewegungspartei-Versuch einerseits und Podemos andererseits verdeutlicht, dass ein prinzipielles Bekenntnis zu radikaldemokratischen Positionen sehr unterschiedlich in die organisatorische Praxis umgesetzt werden kann (vgl. Kim 2019).

\section{Fazit}

Mouffes Plädoyer für einen zur „Radikalisierung der Demokratie“ fähigen Linkspopulismus bildet gewissermaßen die Spitze des Eisbergs einer tieferen Spannungslinie zwischen Populismus und radikaler Demokratie, die sich nicht nur innerhalb des Werks von Laclau und Mouffe, sondern auch an den radikalen Demokratietheorien Leforts und Rancières aufzeigen lässt. Die hier rezipierten Spielarten radikaler Demokratietheorie zeichnen sich allesamt durch Denkfiguren der Kontingenz aus, die wiederum auf die konstitutive Unvollständigkeit der Demokratie und die letztliche Grundlosigkeit des Sozialen verweisen. Während sich dieser Kontingenzgedanke bei Lefort insbesondere in Abgrenzung zur Gefahr des Totalitarismus konstituiert, der einen verabsolutierenden Anspruch auf ein ,Volk' erhebt, verlagert sich die primäre Gefährdung der Kontingenz bei Rancière sowie Mouffe auf die Abwesenheit des ,Volkes“, das wiederum exemplarisch für das ontologisch konstitutive Moment der Teilung bzw. des Antagonismus steht. Im Lichte all dieser theoretischen Spielarten dreht sich aber die konstitutive Spannung zwischen Populismus und radikaler Demokratie grundsätzlich um dasselbe Problem: Dass ein Volkssubjekt gegen die 
herrschenden Machtverhältnisse immer wieder aufs Neue aufgerufen werden kann, gehört konstitutiv zu einem radikalen Demokratieverständnis, verweist aber auch gleichzeitig auf dessen Grenzen, sobald die Anrufung des ,Volkes“ in eine totalisierende Leugnung der Kontingenz (Lefort), in eine neue polizeiliche arche (Rancière) oder in die Beeinträchtigung der Autonomie ,demokratischer Kämpfe“ (Laclau/ Mouffe) umschlägt. In den Verschiebungen von Laclaus und Mouffes radikaler Demokratietheorie zu Laclaus Populismustheorie verdichtet sich eine diskurs- und hegemonietheoretisch greifbare Spannungslinie zwischen Horizontalität und Vertikalität, zwischen Autonomie und Repräsentation: Im Frühwerk Laclaus und Mouffes wird die Autonomie ,demokratischer Kämpfe“ als konstitutive Dimension eines radikaldemokratischen Hegemonieprojekts, in Laclaus späterer Populismustheorie hingegen die repräsentative Funktion von Volkssubjekt und Führung als konstitutiv vorausgesetzt. Damit wird aber auch auf eine allgemeine Spannung zwischen Horizontalität und Vertikalität verwiesen, die im Verhältnis von radikaler Demokratie und Populismus in prototypischer Manier zutage tritt und bei Mouffe wiederum als Frage der organisatorischen Aushandlung akzentuiert wird. In empirisch-analytischer Hinsicht wird damit die Erforschung des Verhältnisses von Populismus und radikaler Demokratie nicht zuletzt zu einer Organisationsfrage, der sich mit einem diskurs- und hegemonietheoretisch fundierten Verständnis von Autonomie und Repräsentation nachgehen lässt - gerade in einem Kontext vermehrter parteiförmiger Versuche, ,vertical structures (party) rooted in horizontalist practices (protests, social movements)“ aufzubauen (Thomassen 2016, S. 173). Als erster Versuch der konzeptuellen Einordnung wurde hier eine Unterscheidung zwischen Bewegungsparteien und Volksparteien neuen Typs als zwei Pole eines Kontinuums zwischen horizontaler Autonomie und vertikaler Repräsentation bzw. Identifikation getroffen, die zur Erfassung eines breiten Universums an organisatorischen Aushandlungen von Horizontalität und Vertikalität beitragen kann und somit einen Ausgangspunkt für weiterführende Forschungen bildet.

Danksagung Der Autor bedankt sich bei Martin Nonhoff, Jakob Schultz und zwei anonymen Gutachter*innen für ihre Kommentare.

Funding Open Access funding enabled and organized by Projekt DEAL.

Open Access Dieser Artikel wird unter der Creative Commons Namensnennung 4.0 International Lizenz veröffentlicht, welche die Nutzung, Vervielfältigung, Bearbeitung, Verbreitung und Wiedergabe in jeglichem Medium und Format erlaubt, sofern Sie den/die ursprünglichen Autor(en) und die Quelle ordnungsgemäß nennen, einen Link zur Creative Commons Lizenz beifügen und angeben, ob Änderungen vorgenommen wurden.

Die in diesem Artikel enthaltenen Bilder und sonstiges Drittmaterial unterliegen ebenfalls der genannten Creative Commons Lizenz, sofern sich aus der Abbildungslegende nichts anderes ergibt. Sofern das betreffende Material nicht unter der genannten Creative Commons Lizenz steht und die betreffende Handlung nicht nach gesetzlichen Vorschriften erlaubt ist, ist für die oben aufgeführten Weiterverwendungen des Materials die Einwilligung des jeweiligen Rechteinhabers einzuholen.

Weitere Details zur Lizenz entnehmen Sie bitte der Lizenzinformation auf http://creativecommons.org/ licenses/by/4.0/deed.de. 


\section{Literatur}

Abts, Koen, und Stefan Rummens. 2007. Populism versus Democracy. Political Studies 55(2):405-424.

Agridopoulos, Aristotelis. 2018. Das anteillose Volk gegen die soziale Hierarchisierung. Zur an-archischen und egalitären Logik im Denken Rancières. In Gleichheit, Politik und Polizei: Jacques Rancière und die Sozialwissenschaften, Hrsg. Thomas Linpinsel, Il-Tschung Lim, 231-248. Wiesbaden: Springer VS.

Canovan, Margaret. 1999. Trust the People! Populism and the Two Faces of Democracy. Political Studies 47(1):2-16.

Canovan, Margaret. 2002. Taking Politics to the People: Populism as the Ideology of Democracy. In Democracies and the Populist Challenge, Hrsg. Yves Mény, Yves Saurel, 25-44. Basingstoke: Palgrave.

Chambers, Samuel. 2011. Jacques Rancière and the Problem of Pure Politics. European Journal of Political Theory 10(3):303-326.

Della Porta, Donatella, et al. 2017. Movement Parties against Austerity. Cambridge: Polity.

Errejón, Íñigo, und Chantal Mouffe. 2015. Construir pueblo. Hegemonía y radicalización de la democracia. Barcelona: Icaria.

Gerbaudo, Paolo. 2017a. The Mask and the Flag: Populism, Citizenism and Global Protest. Oxford: Oxford University Press.

Gerbaudo, Paolo. 2017b. The Indignant Citizen: Anti-Austerity Movements in Southern Europe and the Anti-Oligarchic Reclaiming of Citizenship. Social Movements Studies 16(1):36-50.

Gerbaudo, Paolo. 2018. The Digital Party: Political Organisation and Online Democracy. London: Pluto. Graeber, David. 2012. Inside Occupy. Frankfurt a.M.: Campus.

Hall, Stuart. 1988. The Hard Road to Renewal: Thatcherism and the Crisis of the Left. London: Verso.

Hardt, Michael, und Antonio Negri. 2012. Declaration. New York: Argo-Navis.

Iglesias, Pablo. 2015. Understanding Podemos. New Left Review 93:7-22.

Katsambekis, Giorgos. 2016. Radical Left Populism in Contemporary Greece: Syriza's Trajectory from Minoritarian Opposition to Power. Constellations 23(3):391-403.

Kim, Seongcheol. 2017. The Populism of the Alternative for Germany (AfD): An Extended Essex School Perspective. Palgrave Communications 3(5):1-11.

Kim, Seongcheol. 2019. Radical Democracy and Left Populism after the Squares: Social Movement (Ukraine), Podemos (Spain), and the Question of Organization. Contemporary Political Theory 19(2):211-232.

Kioupkiolis, Alexandros. 2016. Podemos: The Ambiguous Promises of Left-Wing Populism in Contemporary Spain. Journal of Political Ideologies 21(2):99-120.

Kitschelt, Herbert. 1986. Political Opportunity Structures and Political Protest: Anti-Nuclear Movements in Four Democracies. British Journal of Political Science 16(1):57-85.

Kitschelt, Herbert. 2006. Movement Parties. In Handbook of Party Politics, Hrsg. Richard Katz, William Crotty, 278-290. London: SAGE.

Laclau, Ernesto. 1990. New Reflections on the Revolution of Our Time. London: Verso.

Laclau, Ernesto. 2005a. On Populist Reason. London: Verso.

Laclau, Ernesto. 2005b. Populism: What's in a Name? In Populism and the Mirror of Democracy, Hrsg. Francisco Panizza, 32-49. London: Verso.

Laclau, Ernesto. 2005c. The Future of Radical Democracy. In Radical Democracy: Politics Between Abundance and Lack, Hrsg. Lars Tønder, Lasse Thomassen, 256-262. Manchester: Manchester University Press.

Laclau, Ernesto. 2015. An Interview with Ernesto Laclau. In Ernesto Laclau: Post-Marxism, Populism and Critique, Hrsg. David Howarth, 257-271. London: Routledge.

Laclau, Ernesto, und Chantal Mouffe. 2012. Hegemonie und radikale Demokratie. Zur Dekonstruktion des Marxismus. Wien: Passagen.

Lefort, Claude. 1986. The Political Forms of Modern Society: Bureaucracy, Democracy, Totalitarianism. Cambridge: MIT Press.

Lefort, Claude. 1990. Die Frage der Demokratie. In Autonome Gesellschaft und libertäre Demokratie, Hrsg. Ulrich Rödel, 281-297. Frankfurt a.M.: Suhrkamp.

Lefort, Claude. 1992. Écrire: À l'épreuve du politique. Paris: Calmann-Lévy.

Lorey, Isabell. 2012. Demokratie statt Repräsentation. Zur konstituierenden Macht der Besetzungsbewegungen. In Occupy! Die aktuellen Kämpfe um die Besetzung des Politischen, Hrsg. Isabell Lorey, et al., 7-49. Wien: Turia+Kant.

Marchart, Oliver. 2007. Post-Foundational Political Thought: Political Difference in Nancy, Lefort, Badiou and Laclau. Edinburgh: Edinburgh University Press. 
Marchart, Oliver. 2010. Die politische Differenz. Zum Denken des Politischen bei Nancy, Lefort, Badiou, Laclau und Agamben. Berlin: Suhrkamp.

Marchart, Oliver. 2018. Thinking Antagonism: Political Ontology after Laclau. Edinburgh: Edinburgh University Press.

Mayer, Margit, und John Ely (Hrsg.). 1998. The German Greens: Paradox Between Movement and Party. Philadelphia: Temple University Press.

Möller, Kolja. 2017. Invocatio Populi. Autoritärer und demokratischer Populismus. Leviathan 45:246-267.

Mouffe, Chantal. 2017. Über das Politische. Berlin: Suhrkamp.

Mouffe, Chantal. 2018. Für einen linken Populismus. Berlin: Suhrkamp.

Müller, Jan-Werner. 2014. "The People Must Be Extracted from Within the People": Reflections on Populism. Constellations 21(4):483-493.

Müller, Jan-Werner. 2016. Was ist Populismus? Berlin: Suhrkamp.

Nonhoff, Martin. 2019. Ernesto Laclau. In Radikale Demokratietheorie. Ein Handbuch, Hrsg. Dagmar Comtesse, et al., 337-345. Berlin: Suhrkamp.

Prentoulis, Marina, und Lasse Thomassen. 2013. Political Theory in the Square: Protest, Representation and Subjectification. Contemporary Political Theory 12(3):166-184.

Rancière, Jacques. 2002. Das Unvernehmen. Politik und Philosophie. Frankfurt a.M.: Suhrkamp.

Rancière, Jacques. 2008. Zehn Thesen zur Politik. Zürich: diaphanes.

Rancière, Jacques. 2012. Der Hass der Demokratie. Köln: August.

Rancière, Jacques. 2017. Der unauffindbare Populismus. In Was ist ein Volk?, Hrsg. Alain Badiou, et al., 97-101. Hamburg: Laika.

Rendueles, César, und Jorge Sola. 2018. The Rise of Podemos: Promises, Constraints, and Dilemmas. In Podemos and the New Political Cycle: Left-wing Populism and Anti-establishment Politics, Hrsg. Óscar García Augustín, Marco Briziarelli, 25-47. Basingstoke: Palgrave Macmillan.

Richardson, Dick, und Chris Rootes (Hrsg.). 1994. The Green Challenge: The Development of Green Parties in Europe. London: Routledge.

Sitrin, Marina, und Dario Azzellini. 2014. They Can't Represent Us! Reinventing Democracy from Greece to Occupy. London: Verso.

Stavrakakis, Yannis, und Anton Jäger. 2018. Accomplishments and Limitations of the "New" Mainstream in Contemporary Populism Studies. European Journal of Social Theory 21(4):547-565.

Stavrakakis, Yannis, et al, 2017. Extreme Right-Wing Populism in Europe: Revisiting a Reified Association. Critical Discourse Studies 14(3):420-439.

Thomassen, Lasse. 2016. Hegemony, Populism and Democracy: Laclau and Mouffe Today. Revista Española de Ciencia Política 40:161-176.

Tønder, Lars, und Lasse Thomassen. 2005. Introduction: Rethinking Radical Democracy between Abundance and Lack. In Radical Democracy: Politics between Abundance and Lack, Hrsg. Lars Tønder, Lasse Thomassen, 1-13. Manchester: Manchester University Press.

Tsomou, Margarita. 2017. Zwischen Repräsentationskritik, Selbstrepräsentation und nicht-repräsentativen Politiken: die Aktionsformen der Aganaktismenoi auf dem Syntagma-Platz, Athen 2011. Dissertation, HafenCity Universität Hamburg.

Wiesendahl, Elmar, Uwe Jun, und Oskar Niedermayer. 2009. Die Zukunft der Mitgliederparteien auf dem Prüfstand. In Die Zukunft der Mitgliederpartei, Hrsg. Uwe Jun, Oskar Niedermayer, und Elmar Wiesendahl, 9-30. Opladen: Barbara Budrich. 